\title{
Predictive value of De Ritis ratio in metastatic renal cell carcinoma treated with tyrosine-kinase inhibitors
}

\author{
Florian Janisch ${ }^{1,2}$ - Thomas Klotzbücher ${ }^{1}$. Phillip Marks ${ }^{1}$. Christina Kienapfel ${ }^{1}$. Christian P. Meyer ${ }^{1} \cdot$ Hang Yu $^{1}$. \\ Constantin Fühner ${ }^{1} \cdot$ Tobias Hillemacher $^{1} \cdot$ Keiichiro Mori $^{2,3}$. Hadi Mostafei ${ }^{2,4}$. Shahrokh F. Shariat ${ }^{2,5,6,7,8,9}$. \\ Margit Fisch $^{1} \cdot$ Roland Dahlem ${ }^{1} \cdot$ Michael Rink $^{1}$
}

Received: 7 November 2020 / Accepted: 3 February 2021 / Published online: 1 March 2021

(c) The Author(s) 2021

\begin{abstract}
Background Predictive markers can help tailor treatment to the individual in metastatic renal cell carcinoma (mRCC). De Ritis ratio (DRR) is associated with oncologic outcomes in various solid tumors.

Objective To assess the value of DRR in prognosticating survival in mRCC patients treated with tyrosine-kinase inhibitors (TKI).

Methods Overall, $220 \mathrm{mRCC}$ patients treated with TKI first-line therapy were analyzed. An optimal cut-off point for DRR was determined with Youden's J. We used multiple strata for DRR, performed descriptive, Kaplan-Meier and multivariable Cox-regression analyses to assess associations of DRR with progression-free (PFS) and overall survival (OS).

Results Patients above the optimal cut-off point for DRR of $\geq 1.58$ had fewer liver metastases $(p=0.01)$. There was no difference in PFS $(p>0.05)$ between DRR groups. DRR above the median of 1.08 (HR 1.42; $p=0.03$ ), DRR $\geq 1.1$ (HR 1.44; $p=0.02), \geq 1.8($ HR 1.56; $p=0.03), \geq 1.9(\operatorname{HR} 1.59 ; p=0.02)$ and $\geq 2.0$ (HR 1.63; $p=0.047)$ were associated with worse OS. These associations did not remain after multivariable adjustment. In the intermediate MSKCC group, DRR was associated with inferior OS at cut-offs $\geq 1.0$ (HR 1.78; $p=0.02), \geq 1.1$ (HR 1.81; $p=0.01)$ and above median (HR 1.88; $p=0.007)$ in multivariable analyses. In patients with clear-cell histology, DRR above median (HR $1.54 ; p=0.029)$ and DRR $\geq 1.1$ (HR $1.53 ; p=0.029$ ) were associated with OS in multivariable analyses.

Conclusion There was no independent association between DRR and survival of mRCC patients treated with TKI in the entire cohort. However, OS of patients with intermediate risk and clear-cell histology were affected by DRR. DRR could be used for tailored decision-making in these subgroups.
\end{abstract}

Keywords Metastatic renal cell carcinoma $\cdot$ Tyrosine-kinase inhibitors $\cdot$ Tumor markers $\cdot$ Prognostic marker $\cdot$ De ritis ratio

Florian Janisch and Thomas Klotzbücher contributed equally to this manuscript.

Roland Dahlem and Michael Rink contributed equally to this work.

Michael Rink

m.rink@uke.de

1 Department of Urology, University Medical Center Hamburg-Eppendorf, Martinistraße 52, 20246 Hamburg, Germany

2 Department of Urology, Medical University of Vienna, Vienna, Austria

3 Department of Urology, Jikei University School of Medicine, Tokyo, Japan

4 Department of Urology, Tabriz University of Medical Sciences, Tabriz, Iran
5 Institute for Urology and Reproductive Health, Sechenov University, Moscow, Russia

6 Department of Urology, Weill Cornell Medical School, New York, NY, USA

7 Department of Urology, University of Texas Southwestern Medical Center, Dallas, TX, USA

8 Karl Landsteiner Institute of Urology and Andrology, Vienna, Austria

9 Department of Urology, Second Faculty of Medicine, Charles University, Prague, Czech Republic 


\section{Introduction}

Despite the progress in the therapy of metastatic renal cell carcinoma (mRCC), including the introduction of tyrosinekinase inhibitors (TKI), survival rates for $\mathrm{mRCC}$ remain low with $<20 \%$ of patients surviving beyond 5 years from their diagnosis [1]. Currently used prognostic risk stratifications such as the Memorial Sloan Kettering Cancer Center (MSKCC) and the International Metastatic RCC Database Consortium (IMDC) [2,3] have great value in identifying patients' prognosis. However, the rapid evolution of systemic treatment and surge of combination therapies requires further individualized refinement of treatment to provide optimal treatment for $\mathrm{mRCC}$ patients.

To address this difficulty, several prognostic markers have been investigated to help daily clinical decisionmaking and tailoring treatment to the individual $[4,5]$. However, as of now, none of the investigated biomarkers pass from bench-to-bedside [6].

Inflammatory markers have been suggested to provide important information about the function of our body's immune system with carcinomas. Multiple actors in the immune system such as the C-reactive protein and neutrophil-to-lymphocyte ratio have been shown to be of prognostic relevance in mRCC [7]. The De Ritis ratio (DRR), the ratio between the serum concentrations of aspartate transaminase (AST) and alanine transaminase (ALT), demonstrated to predict survival outcomes in hepatic disease as well as other solid organ malignancies $[8,9]$. Recent reports found the DRR to be of value in patients with localized RCC [10]. DRR is an easily assessable and cheap marker that could prove valuable in the prognostication of mRCC. However, specific cut-off points for DRR are not well defined and possibly differ from disease state, type and population case mix.

We hypothesized that the DRR is a prognosticator for oncologic outcomes in patients with mRCC. The aim of this study is to analyze the association between DRR and different survival endpoints in a consecutive, real-world cohort of mRCC patients treated with TKI therapy.

\section{Materials and methods}

\section{Patient selection}

We retrospectively reviewed the records of 398 consecutive patients with $\mathrm{mRCC}$ treated at our institution from 2006 to 2016. Only patients with primary TKI therapy with or without cytoreductive nephrectomy were included in this study. Patients with prior immunotherapy, missing follow-up, clinical information or laboratory values, or proven liver disease prior to treatment were excluded $(n=178)$. This left a total of 220 patients for analysis. Data for baseline characteristics and clinical outcome including age, gender, Eastern Cooperative Oncology Group performance status (ECOG), underlying primary histology, pathological T-stage, nodal status, details about performed cytoreductive nephrectomy, number and locations of metastasis, presence of secondary malignancy in patient's history, metastasectomy and therapy lines were gathered. The MSKCC risk score was calculated for each patient [3]. ECOG status, MSKCC and Karnofsky score were assessed before treatment. ALT and AST were assessed before initiation of systemic therapy or cytoreductive nephrectomy. Cut-off of metastatic locations was $\leq 2 \mathrm{vs}$. $\geq 3$ metastatic sites, as previously described [11]. The study was approved by the institutional review board.

\section{Histological assessment}

Surgical specimens were processed according to standard pathologic procedures. Tumor stage and grade were classified according to the American Joint Committee on Cancer-Union Internationale Contre le Cancer TNM classification and the $1998 \mathrm{WHO} /$ International Society of Urologic Pathology consensus classification. Incomplete pathological stage or nodal status due to specimen taken from biopsies was categorized as pTx/pNx. Primary histology was assessed by a dedicated uro-pathologist according to the World Health Organisation (WHO) histologic classification. Histology was grouped according to subtypes in clear-cell, papillary, chromophobe and "other" RCC, respectively [12]. Sarcomatoid histology was assessed from the primary tumor or biopsy, as previously described [13].

\section{Follow-up}

Patients were regularly seen in our outpatient clinic and the follow-up was performed according to the current guidelines at the time [11]. Clinical examination and laboratory controls were performed monthly. Diagnostic imaging of the abdomen and pelvis as well as chest radiography were conducted quarterly. Additional radiographic evaluations (e.g., bone or brain imaging) were performed when clinically indicated. Primary co-endpoints were progression-free (PFS) and overall survival (OS). Disease progression was defined by clinical progression according to the current Response Evaluation Criteria in Solid Tumors (RECIST) version at the time of evaluation [14]. All patients without disease progression or death at last follow-up were censored. 


\section{Statistical analysis}

Continuous variables were reported as mean and standard error when normal distributed, or as median and interquartile range. DRR score was calculated as AST divided by ALT. Youden's J statistic was used to determine the optimal cut-off point for the DRR ratio with a receiver-operator curve using median cancer-specific survival as an endpoint $[15,16]$. In addition, the DRR ratio was analyzed as a continuous variable, stratified at the median, tertiles and according to cut-off points from 1.0 to 2.0 in 0.1 steps to account for possible intervariability of study cohorts in the current literature and provide the most complete evidence. Restricted cubic splines were used to visualize the relation between DRR and the probability of death [17]. Baseline characteristics were compared using the Chi-square test or Fisher's exact test for nominal variables and Student's $T$ test or Mann-Whitney $U$ test for continuous variables. Survival analysis was performed with Kaplan-Meier estimates using the log-rank test for pairwise comparison. Uni- and multi-variable analyses were performed with a Cox-regression model, adjusting for the effect of clinically relevant factors. We performed a competing risk analysis using cancer-specific and other-cause mortality. All analyses were performed in STATA 14.0 (Stata Corp., College Station, TX). Statistical results were considered significant if the $p$ value was $<0.05$; all tests were two sided.

\section{Results}

\section{Baseline characteristics}

Youden's J revealed an optimal cut-off point for DRR at 1.58 (supplementary Fig. 1); patients were grouped accordingly into "low" and "high DRR", and baseline characteristics are displayed in Table 1 accordingly. Overall, 171 patients (77.7\%) had a low DRR and $49(22.3 \%)$ had a high DRR. Median AST was 22 IU/L (IQR 17;33 IU/L) and median ALT was $21 \mathrm{IU} / \mathrm{L}$ (IQR 14;32 IU/L) resulting in a median DRR of 1.08 (IQR 0.81-1.50). Median age was 64 years (IQR 57;71) and 163 patients $(74.1 \%)$ were male. Most patients had clear-cell histology $(n=182 ; 82.7 \%)$ and received Sunitinib as first-line treatment $(n=153 ; 69.6 \%)$, respectively. 157 (71.4\%) patients had $\geq 3$ metastatic sites and $73(33.2 \%)$ had liver metastases. Patients with high DRR had less frequent liver metastasis compared to those with low DRR $(p=0.01)$, but no difference in the number of metastatic sites. There were no differences in other baseline characteristics between the two groups.

\section{Association of DRR with survival outcomes}

The median follow-up was 72 months (IQR 42;126), median PFS was 13 months (IQR 4;38) and median OS was 28 months (IQR 10;58). Median observed and expected mortalities in relation to DRR are presented in supplementary Fig. 2 with the probability of death rising from a low DRR to 1.8 and a falling with a low decrement at higher levels. Kaplan-Meier estimates did not reveal a difference in PFS between patients with high or low DRR (Fig. 1a). There was also no association between DRR and PFS for all analyzed strata (all $p>0.05$; Table 2).

Patients with low DRR had better OS in Kaplan-Meier estimates (35 vs. 22 months), which was not statistically significant $(p=0.08$; Fig. 1b). A high DRR, continuous DRR, and DRR stratified by tertiles (cut-off points: 0.89 and 1.36) were not significantly associated with OS in univariable analyses (Table 2). Patients with DRR above the median compared to those with DRR below median (HR $1.42 ; 95 \% \mathrm{CI}=1.04-1.93 ; p=0.03)$, and all DRR above the threshold of 1.1 (HR 1.44; 95\% CI $=1.06-1.96 ; p=0.02)$, 1.8 (HR 1.56; 95\% CI = 1.05-2.31; $p=0.03), 1.9$ (HR 1.59; $95 \% \mathrm{CI}=1.06-1.96 ; p=0.03)$ and $2.0(\mathrm{HR} 1.63 ; 95 \%$ $\mathrm{CI}=1.01-2.64 ; p=0.047)$ were associated with worse OS compared to patients below these cut-off points (Table 2). These associations, however, did not remain significant in multivariable analyses that adjusted for standard outcome mRCC parameters (all $p>0.05$, Table 2). All other cut-off points for DRR did not show any association with survival outcomes in univariable analyses. Competing risk analysis showed no difference between high DRR and low DRR for cancer-specific and other-cause mortality (Supplementary Fig. $3 ; p>0.05)$.

\section{Subgroup analyses of prognostic risk groups}

The results of subgroup analyses are presented in supplementary Table 1 and Fig. 1c, d. In patients with good prognosis, there was no association between all strata for DRR and PFS or OS in multivariable analyses (all $p>0.05$ ). In the subgroup of patients with intermediate prognosis, PFS was not associated with DRR (all $p>0.05$ ). DRR higher than the cut-off points of $1.0(\mathrm{HR} 1.78 ; 95 \% \mathrm{CI}=1.09-2.90 ; p=0.02)$ and 1.1 (HR $1.81 ; 95 \% \mathrm{CI}=1.15-2.85 ; p=0.01)$, as well as the median (HR $1.88 ; 95 \% \mathrm{CI}=1.19-2.96 ; p=0.007$ ) were associated with inferior OS in multivariable analyses in patients with intermediate prognosis. All other cut-off points for DRR did not show any association with survival outcomes in univariable analyses. In poor prognosis patients, PFS was only associated with continuous DRR (HR 0.63; $95 \% \mathrm{CI}=0.39-1.00 ; p=0.049)$ in univariable analysis. This effect, however, did not retain in multivariable analysis 
Table 1 Baseline characteristics of patients with metastatic renal cell carcinoma treated with tyrosine-kinase inhibitors stratified by DRR

\begin{tabular}{|c|c|c|c|c|}
\hline n $(\%)$ & $\begin{array}{l}\text { Overall } \\
220\end{array}$ & $\begin{array}{l}\text { Low DRR } \\
171(77.7)\end{array}$ & $\begin{array}{l}\text { High DRR } \\
49(22.3)\end{array}$ & $p$ value \\
\hline Age—median (IQR) & $64(57-71)$ & $64(57-71)$ & $63(59-70)$ & 0.8 \\
\hline Male gender & $163(74.1)$ & $126(73.7)$ & $31(75.1)$ & 0.06 \\
\hline $\mathrm{ECOG} \geq 2$ & $27(12.3)$ & $20(11.8)$ & $7(14.3)$ & 0.63 \\
\hline Karnofsky $>80 \%$ & $152(69.4)$ & $123(72.4)$ & $29(59.2)$ & 0.08 \\
\hline Histology & & & & 0.6 \\
\hline Clear cell & $182(82.7)$ & 143 (83.6) & 39 (79.6) & \\
\hline Papillary & $28(12.7)$ & $22(12.9)$ & $6(12.2)$ & \\
\hline Chromophobe & $7(3.2)$ & $4(2.3)$ & $3(6.1)$ & \\
\hline Other & $3(1.4)$ & $2(1.2)$ & $1(2.0)$ & \\
\hline Variant histology & $18(8.2)$ & $15(8.8)$ & $3(6.1)$ & 0.6 \\
\hline Sarcomatoid component & $16(7.3)$ & $14(8.2)$ & $2(4.1)$ & 0.3 \\
\hline pT stage & & & & 0.6 \\
\hline pT1 & $51(23.2)$ & $42(24.6)$ & $9(18.4)$ & \\
\hline pT2 & $35(15.9)$ & $28(16.4)$ & $7(14.3)$ & \\
\hline pT3 & $92(41.8)$ & $72(42.1)$ & $20(40.8)$ & \\
\hline pT4 & $14(6.4)$ & $10(5.9)$ & $4(8.2)$ & \\
\hline $\mathrm{pTx}^{\mathrm{a}}$ & $28(12.7)$ & $19(11.1)$ & $9(18.4)$ & \\
\hline Nodal status & & & & 0.4 \\
\hline pN0 & 79 (35.9) & $65(38.0)$ & $14(28.6)$ & \\
\hline $\mathrm{pN}+$ & 43(19.6) & $31(18.3)$ & $23(46.9)$ & \\
\hline $\mathrm{pNx}^{\mathrm{a}}$ & $98(44.5)$ & $75(43.9)$ & $12(24.5)$ & \\
\hline MSKCC score & & & & 0.4 \\
\hline Good & $47(21.4)$ & $36(21.1)$ & $11(22.5)$ & \\
\hline Intermediate & $128(58.2)$ & $103(60.2)$ & $25(51.0)$ & \\
\hline Poor & $45(20.5)$ & $32(18.7)$ & $13(26.5)$ & \\
\hline Secondary malignancy & $35(15.9)$ & $24(14.0)$ & $11(22.5)$ & 0.16 \\
\hline AST—median (IQR) & $22(17-33)$ & $22(17-32)$ & $24(18-33)$ & 0.6 \\
\hline ALT—median (IQR) & $21(14-32)$ & $24(17-38)$ & $12(9-18)$ & $<0.001$ \\
\hline DRR—median (IQR) & $1.08(0.81-1.50)$ & $0.94(0.73-1.21)$ & $1.92(1.75-2.12)$ & $<0.001$ \\
\hline \multicolumn{5}{|l|}{ Metastasis locations } \\
\hline Brain & $33(15.0)$ & $23(13.5)$ & $10(20.4)$ & 0.2 \\
\hline Bone & $113(51.4)$ & $84(59.1)$ & $29(59.2)$ & 0.2 \\
\hline Lung & $162(73.6)$ & $124(72.5)$ & $38(77.6)$ & 0.5 \\
\hline Liver & $73(33.2)$ & $64(37.4)$ & $9(18.4)$ & 0.01 \\
\hline Lymph nodes & $163(74.1)$ & $124(72.5)$ & $39(79.6)$ & 0.3 \\
\hline Other & $127(57.7)$ & $99(57.9)$ & $28(57.1)$ & 0.9 \\
\hline$\geq 3$ metastastic sites & $157(71.4)$ & $125(73.1)$ & $32(65.3)$ & 0.3 \\
\hline Cytoreductive nephrecotmy & $83(37.7)$ & $65(38.0)$ & $18(36.7)$ & 0.9 \\
\hline Metastasectomy & $114(51.8)$ & $86(50.3)$ & $28(57.1)$ & 0.4 \\
\hline First-line therapy & & & & 0.4 \\
\hline Suntinib & $153(69.6)$ & $122(71.4)$ & $31(63.3)$ & \\
\hline Sorafenib & $29(13.1)$ & $20(11.7)$ & $5(10.2)$ & \\
\hline Pazopanib & $27(12.3)$ & $22(12.9)$ & $9(18.4)$ & \\
\hline Other & $11(5.0)$ & $7(4.1)$ & $4(8.2)$ & \\
\hline$\geq 3$ therapy lines & $63(28.6)$ & $53(31.0)$ & $10(20.4)$ & 0.1 \\
\hline
\end{tabular}

Bold values indicate significant $(p<0.05)$

$A L T$ alanine transaminase, AST aspartate transaminase, DRR De Ritis ratio (AST/ALT), ECOG Eastern Cooperative Oncology Group performance status, MSKCC Memorial Sloan Kettering Cancer Center, IQR interquartile range, $p T$ stage pathological tumor stage

${ }^{a}$ Pathological nodal status / T stage not available in patients with biopsies only

${ }^{\mathrm{b}}$ Cut-off point for high and low De Ritis ratio 1.58 

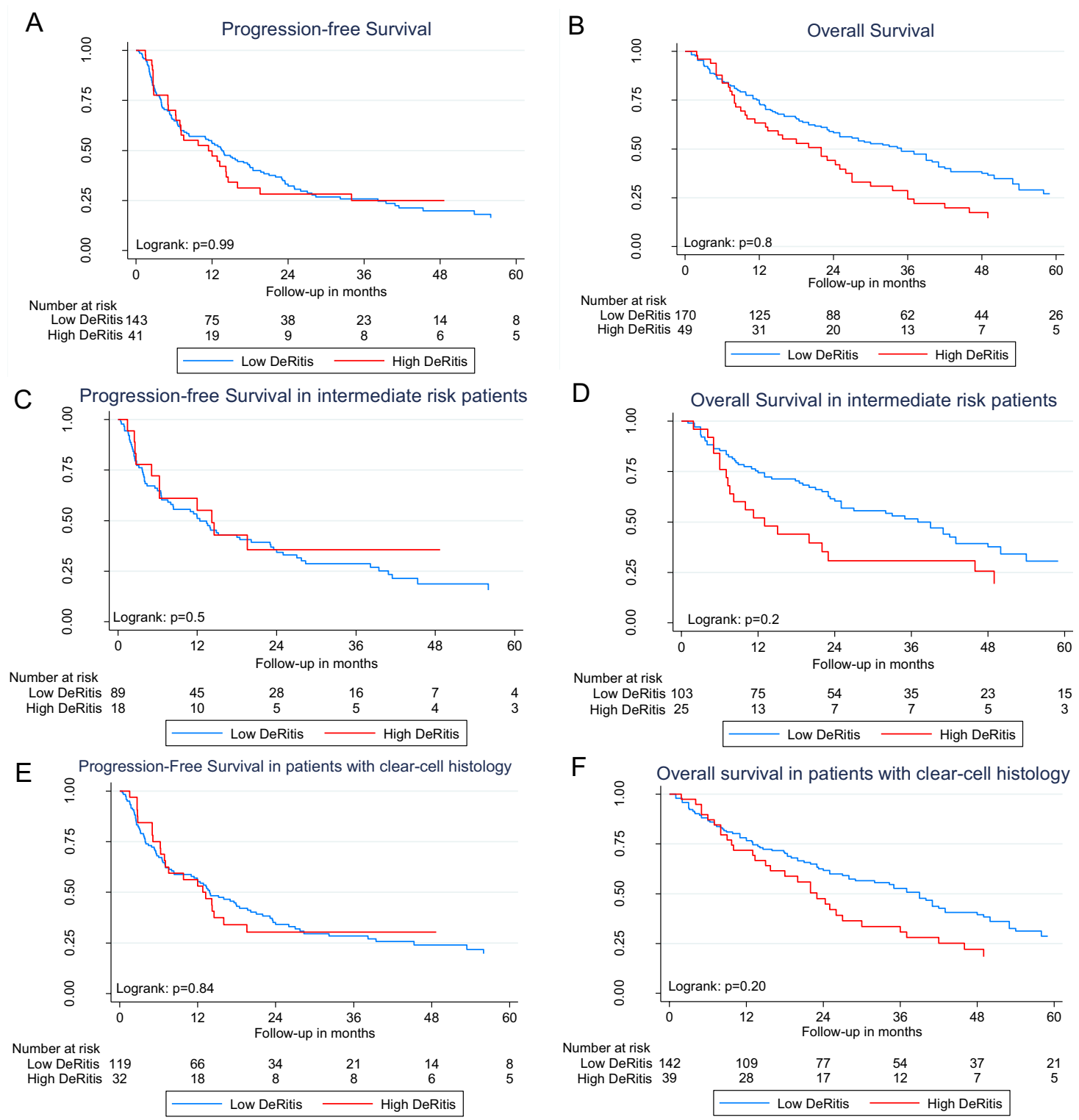

Fig. 1 Kaplan-Meier estimates of progression-free (a) and overall survival (b) of patients with metastatic renal cell carcinoma and the subgroups of intermediate-risk patients (progression-free survival;

$(p>0.05)$. There was no association between DRR and OS in poor prognosis patients.

\section{Subgroup analyses of patients with clear-cell histology}

Supplementary Table 2 and Fig. 1e, f show the results from the subgroup analyses of patients with and without clear-cell histology. In patients with clear-cell histology,

c) (overall survival; d) and clear-cell histology (progression-free survival; e) (overall survival; f) stratified by a low $(<1.58)$ and high $(\geq 1.58)$ De Ritis ratio

there was no association between PFS and DRR. Patients with DRR higher than the cut-off points 1.1 (HR 1.53; 95\% $\mathrm{CI}=1.04-2.24 ; p=0.029)$ as well as the median (HR $1.54 ; 95 \% \mathrm{CI}=1.05-2.29 ; p=0.029$ ) had shorter OS compared to those below these thresholds in multivariable analyses. There was no association between either PFS or OS and DRR in patients with non-clear-cell histology (all $p>0.05)$. 
Table 2 Uni- and multi-variable regression analyses of different strata for De Ritis ratio (DRR) and its impact on progression-free (PFS) and overall survival (OS)

\begin{tabular}{|c|c|c|c|c|c|c|c|c|c|}
\hline & \multicolumn{3}{|c|}{ Univariable PFS } & \multicolumn{3}{|c|}{ Univariable OS } & \multicolumn{3}{|c|}{ Multivariable OS } \\
\hline & HR & $95 \% \mathrm{CI}$ & $p$ value & HR & $95 \% \mathrm{CI}$ & $p$ value & HR & $95 \% \mathrm{CI}$ & $p$ value \\
\hline DRR (continuous) & 1.06 & $0.84-1.33$ & 0.64 & 1.22 & $0.98-1.52$ & 0.08 & - & - & - \\
\hline High vs. low $\mathrm{DRR}^{\mathrm{a}}$ & 1.00 & $0.67-1.50$ & 0.99 & 1.37 & $0.96-1.96$ & 0.08 & - & - & - \\
\hline $\begin{array}{l}\mathrm{DRR} \geq \text { median vs. }<\text { median } \\
\text { DRR tertiles }\end{array}$ & 1.11 & $0.80-1.55$ & 0.54 & 1.42 & $1.04-1.93$ & 0.03 & 1.37 & $0.99-1.93$ & 0.07 \\
\hline 2 nd vs. $1 \mathrm{st}$ & 1.33 & $0.88-2.02$ & 0.18 & 1.21 & $0.92-1.77$ & 0.34 & - & - & - \\
\hline 3rd vs. 1st & 1.16 & $0.77-1.75$ & 0.48 & 1.31 & $0.90-1.91$ & 0.16 & - & - & - \\
\hline $\mathrm{DRR} \geq 1.1$ vs. $<1.1$ & 1.05 & $0.75-1.46$ & 0.79 & 1.44 & $1.06-1.96$ & 0.02 & 1.36 & $0.97-1.90$ & 0.07 \\
\hline DRR $\geq 1.8$ vs. $<1.8$ & 1.18 & $0.75-2.87$ & 0.47 & 1.56 & $1.05-2.31$ & $\mathbf{0 . 0 3}$ & 1.49 & $0.99-2.26$ & 0.056 \\
\hline DRR $\geq 1.9$ vs. $<1.9$ & 1.25 & $0.76-2.06$ & 0.38 & 1.59 & $1.04-2.44$ & 0.03 & 1.37 & $0.87-2.15$ & 0.18 \\
\hline DRR $\geq 2.0$ vs. $<2.0$ & 1.29 & $0.73-2.29$ & 0.39 & 1.63 & $1.01-2.64$ & 0.047 & 1.30 & $0.78-2.17$ & 0.31 \\
\hline
\end{tabular}

Bold values indicate significant $(p<0.05)$

All DRR cut-off points that were not significant in the univariable analysis are not shown

Multivariable analysis adjusted for significant values in the univariable analysis of the following: Eastern Cooperative Oncology Group performance status (ECOG), histology subgroup and sarcomatoid features, Memorial Sloan Kettering Cancer Center prognostic risk score (MSKCC), number of therapy lines, presence of sarcomatoid histology, secondary malignancy, $\mathrm{T}$ stage and lymph node metastasis, presence of liver metastasis and number of metastatic locations

Adjustments in multivariable analyses were performed for ECOG histology subgroup and sarcomatoid features, T stage and lymph node metastasis, MSKCC, and number of therapy lines for PFS and for ECOG, sarcomatoid histology, cytoreductive nephrectomy, pathological T stage and lymph node metastasis, MSKCC for OS, respectively

${ }^{a}$ Cut-off point for high and low De Ritis ratio 1.58

\section{Discussion}

In this study, we analyzed the association between DRR and oncologic outcomes in mRCC patients treated with TKI. The cut-off point determined by Youden's J could not prove more predictive for survival in comparison to other strata. We found no difference in PFS or OS in patients with higher DRR, regardless of cut-off points or stratification method. Nonetheless, competing risk analysis did not show any difference in cancer-specific survival in patients with high DRR. Thus, our findings challenge the value of DRR as a prognostic marker in $\mathrm{mRCC}$ patients.

Indeed, our results are in contrast to some body of literature. Kang et al. reported better OS and CSS with DRR $<1.2$ before TKI therapy initiation [18]. Their patients with higher DRR, however, were significantly older, and $>20 \%$ of underlying histology and pathological $\mathrm{T}$ stage was missing from their dataset, which possibly skewed results. Kim et al. found higher continuous DRR to be an independent factor for worse OS, but not PFS in patients with mRCC [19]. However, the study included patients with missing AST or ALT as well, possibly introducing bias. Another study reported that DRR $\geq 1.24$ was associated with worse OS and CSS in patients with $\mathrm{mRCC}$ that underwent cytoreductive nephrectomy [20]. In a recent meta-analysis, DRR was associated with survival in patients with RCC [8]. However, the heterogeneous designs of the included studies, including localized and metastatic disease as well as different cut-off points, impair comparability. Interestingly, we found that patients with clear-cell histology had worse OS when DRR was $\geq 1.1$ or the median of 1.08 . Patients with other, varied underlying histologic subtypes might have skewed results in the entire cohort. In contrast, Sekar et al. found no difference in OS in their subgroup analyses of patients with clear-cell histology [21]. However, this study included all patients with performed nephrectomy and included patients with localized and metastatic disease. This further suggests that heterogeneity in study cohorts should be minimized to shed light on optimal cut-off points for DRR and help finding suitable patient subgroups to incorporate this marker into clinical treatment. Thus, our findings are of utmost importance as the true value of DRR in $\mathrm{mRCC}$ should be reevaluated in larger and ideally prospective studies.

We found that patients in the MSKCC intermediate-risk group with DRR lower than the cut-off point 1.0-1.1 had improved OS, while outcomes were not influenced in other risk groups in multivariable analysis. These findings warrant consideration, as prognostic risk group scores are standard tools in $\mathrm{mRCC}$ for patient stratification to facilitate optimal systemic treatment. However, particularly the intermediaterisk group, which comprises the largest number of patients, is challenging regarding treatment and prognosis, due to its heterogeneity. Indeed, differential survival outcomes in this risk group are highly dependent on the number of 
risk factors [22]. Patients with only one risk factor in the intermediate-risk group according to IMDC score had a 13-month-longer median OS in mRCC patients treated with sunitinib [23]. This underscores the necessity of differential assessment in the intermediate-risk group. Further, insignificant results of the good and poor prognosis groups should be validated, to overcome potential impairment by small sample sizes. Incorporation of DRR into established risk scores may have the potential to tailor patient counseling and help adjust follow-up regimen. Additional research in larger, prospective studies is recommended, as the current body of literature remains inconclusive and our results are just hypothesis generating $[19,21]$.

One of the first studies to describe the impact of DRR on survival outcomes in cancer patients is the study of Bezan et al., which focused on patients with localized RCC [24]. Since then, several other studies investigated the association of DRR and survival in localized RCC with conflicting results $[10,25]$. Indeed, in mRCC, the metastatic burden predominantly impacts survival. Visceral metastases, especially liver metastases, are associated with poor oncologic outcomes [26]. High transaminase levels may be used as a surrogate parameter for liver metastasis (i.e., liver damage), and, in consequence, for oncologic outcomes. Therefore, DRR could possibly present an interesting marker depending on metastatic location patterns in contrast to other prognosticators. Albeit, the number of metastatic sites had no association with high DRR in our study. Interestingly, hepatic metastases were even less frequent in patients with a high DRR. To avoid bias, we calculated DRR based on pretreatment laboratory values, as transaminase levels may be influenced by systemic therapy. Still, the value of this score should be reevaluated during the course of treatment, as visceral metastases may develop during disease progression.

Our study is not without limitations. First, the retrospective single-center design introduces patient selection bias. Second, as not all included patients underwent nephrectomy at any time, information about pathological tumor stage and nodal status was not available for all patients, which may have influenced statistical analyses. Third, metastatic burden was assessed by imaging and not always verified by biopsy. Fourth, DRR is an unspecific marker that could be affected by other comorbidities and drugs as well, despite defining clear in- and exclusion criteria. Additionally, not all comorbidities and corresponding medication were present in our data set in detail, and thus could not be included, which might have influenced our findings. Meanwhile, new drugs have progressed to clinical standard in mRCC [27] and mainly superseded those in our cohort. However, TKI used in our study were standard of care at the time of treatment and, therefore, should not bias outcomes. In addition, TKI still remains an important treatment option in selected patients. Reevaluation of DRR in current treatment regimens including immuno-oncology (IO)/TKI or IO/IO combinations is warranted. Further, a single measurement of DRR before treatment cannot solely portray the dynamics in each patient's metabolism during systemic therapy. As TKI therapy can result in the elevation of liver enzyme levels, DRR might not be a fitting prognosticator for further therapy lines. While setting a standardized point is necessary for accurate statistics, the prognostic value of DRR might change during systemic therapy. However, we feel that the variable strata used to define cut-off points in our study provided valuable insight on DRR as a prognostic marker.

\section{Conclusion}

There was no strong association between various DRR strata and survival outcomes in our entire mRCC cohort. However, DRR may help in patient prognostication in the large, heterogeneous MSKCC intermediate-risk subgroup as well as in patients with clear-cell histology. Thus, the incorporation of DRR in clinical decision-making and treatment guidance of intermediate-risk patients in our western European population warrants further investigation. Additional, larger, prospective studies are needed to further assess the value of DRR in other western-world regions and address potential confounders.

Supplementary Information The online version contains supplementary material available at https://doi.org/10.1007/s00345-021-03628-2.

Author contributions FJ: protocol/project development, data analysis, and manuscript writing/editing. TK: data collection or management. PM: manuscript writing/editing, data collection or management. CK: data collection or management. CPM: protocol/project development and manuscript writing/editing. HY: data collection or management. $\mathrm{CF}$ : manuscript writing/editing. $\mathrm{TH}$ : data collection or management. KM: data analysis. HM: data analysis. SFS: protocol/project development. MF: protocol/project development and manuscript writing/editing. RD: protocol/project development and manuscript writing/editing. MR: protocol/project development and manuscript writing/editing.

Funding Open Access funding enabled and organized by Projekt DEAL.

\section{Compliance with ethical standards}

Conflict of interest Prof. Sharokh F. Shariat is consulting or advising the following: Astra Zeneca, BMS, Ferring, Ipsen, Jansen, MSD, Olympus, Pfizer, Pierre Fabre, Richard Wolf, Roche, Sanochemia, and Urogen. Dr. Rink is a speaker for Bayer Healthcare, Bristol Myer Squibb, EUSA Pharma, IPSEN Pharma, Novartis, Roche, and Pfizer. Dr. Rink is a consultant and/or received honoraria by Bayer Healthcare, Bristol Myer Squibb, IPSEN Pharma, MSD, Novartis, Roche, and Pfizer. The other authors do not have conflicts of interest present. 
Ethical statement The authors are accountable for all aspects of the work in ensuring that questions related to the accuracy or integrity of any part of the work are appropriately investigated and resolved. All procedures performed in studies involving human participants were in accordance with the ethical standards of the institutional research committee and with the 1964 Helsinki Declaration and its later amendments or comparable ethical standards.

Informed consent Informed consent was obtained from all individual participants included in the study.

Open Access This article is licensed under a Creative Commons Attribution 4.0 International License, which permits use, sharing, adaptation, distribution and reproduction in any medium or format, as long as you give appropriate credit to the original author(s) and the source, provide a link to the Creative Commons licence, and indicate if changes were made. The images or other third party material in this article are included in the article's Creative Commons licence, unless indicated otherwise in a credit line to the material. If material is not included in the article's Creative Commons licence and your intended use is not permitted by statutory regulation or exceeds the permitted use, you will need to obtain permission directly from the copyright holder. To view a copy of this licence, visit http://creativecommons.org/licenses/by/4.0/.

\section{References}

1. Keizman D, Huang P, Eisenberger MA, Pili R, Kim JJ, Antonarakis ES et al (2011) Angiotensin system inhibitors and outcome of sunitinib treatment in patients with metastatic renal cell carcinoma: a retrospective examination. Eur J Cancer 47(13):19551961. https://doi.org/10.1016/j.ejca.2011.04.019

2. Heng DY, Xie W, Regan MM, Warren MA, Golshayan AR, Sahi C et al (2009) Prognostic factors for overall survival in patients with metastatic renal cell carcinoma treated with vascular endothelial growth factor-targeted agents: results from a large, multicenter study. J Clin Oncol 27(34):5794-5799. https://doi.org/10.1200/ jco.2008.21.4809

3. Motzer RJ, Bacik J, Murphy BA, Russo P, Mazumdar M (2002) Interferon-alfa as a comparative treatment for clinical trials of new therapies against advanced renal cell carcinoma. J Clin Oncol 20(1):289-296. https://doi.org/10.1200/jco.2002.20.1.289

4. Rink M, Chun FK, Robinson B, Sun M, Karakiewicz PI, Bensalah $\mathrm{K}$ et al (2011) Tissue-based molecular markers for renal cell carcinoma. Minerva Urol Nefrol 63(4):293-308

5. Li H, Samawi H, Heng DY (2015) The use of prognostic factors in metastatic renal cell carcinoma. Urol Oncol 33(12):509-516. https://doi.org/10.1016/j.urolonc.2015.08.003

6. Bensalah K, Montorsi F, Shariat SF (2007) Challenges of cancer biomarker profiling. Eur Urol 52(6):1601-1609. https://doi. org/10.1016/j.eururo.2007.09.036

7. Ito K, Masunaga A, Tanaka N, Mizuno R, Shirotake S, Yasumizu $\mathrm{Y}$ et al (2019) Impact of inflammatory marker levels one month after the first-line targeted therapy initiation on progression-free survival prediction in patients with metastatic clear cell renal cell carcinoma. Jpn J Clin Oncol 49(1):69-76. https://doi.org/10.1093/ jjco/hyy 154

8. Wu J, Chen L, Wang Y, Tan W, Huang Z (2019) Prognostic value of aspartate transaminase to alanine transaminase (De Ritis) ratio in solid tumors: a pooled analysis of 9400 patients. Oncol Targets Ther 12:5201-5213. https://doi.org/10.2147/OTT.S204403

9. Deritis F, Giusti G, Piccinino F, Cacciatore L (1965) Biochemical laboratory tests in viral hepatitis and other hepatic diseases. Evaluation and follow-uP. Bull World Health Organiz 32(1):59-72
10. Lee H, Lee SE, Byun SS, Kim HH, Kwak C, Hong SK (2017) De Ritis ratio (aspartate transaminase/alanine transaminase ratio) as a significant prognostic factor after surgical treatment in patients with clear-cell localized renal cell carcinoma: a propensity scorematched study. BJU Int 119(2):261-267. https://doi.org/10.1111/ bju. 13545

11. Janisch F, Hillemacher T, Fuehner C, D'Andrea D, Meyer CP, Klotzbücher T et al (2020) The impact of cytoreductive nephrectomy on survival outcomes in patients treated with tyrosine kinase inhibitors for metastatic renal cell carcinoma in a real-world cohort. Urol Oncol. https://doi.org/10.1016/j.urolonc.2020.04.033

12. Capitanio U, Cloutier V, Zini L, Isbarn H, Jeldres C, Shariat SF et al (2009) A critical assessment of the prognostic value of clear cell, papillary and chromophobe histological subtypes in renal cell carcinoma: a population-based study. BJU Int 103(11):14961500. https://doi.org/10.1111/j.1464-410X.2008.08259.x

13. Janisch F, Kienapfel C, Fühner C, Klotzbücher T, Marks P, Hillemacher $\mathrm{T}$ et al (2020) Treatment and outcome of metastatic renal cell carcinoma with sarcomatoid differentiation. Currently under review

14. Therasse P, Arbuck SG, Eisenhauer EA, Wanders J, Kaplan RS, Rubinstein L et al (2000) New guidelines to evaluate the response to treatment in solid tumors. European Organization for Research and Treatment of Cancer, National Cancer Institute of the United States, National Cancer Institute of Canada. J Natl Cancer Inst 92(3):205-216. https://doi.org/10.1093/jnci/92.3.205

15. Schisterman EF, Perkins NJ, Liu A, Bondell H (2005) Optimal cut-point and its corresponding Youden Index to discriminate individuals using pooled blood samples. Epidemiology 16(1):73-81

16. Heagerty PJ, Lumley T, Pepe MS (2000) Time-dependent ROC curves for censored survival data and a diagnostic marker. Biometrics 56(2):337-344. https://doi.org/10.1111/j.0006341x.2000.00337.x

17. Durrleman S, Simon R (1989) Flexible regression models with cubic splines. Stat Med 8(5):551-561. https://doi.org/10.1002/ sim.4780080504

18. Kang M, Yu J, Sung HH, Jeon HG, Jeong BC, Park SH et al (2018) Prognostic impact of the pretreatment aspartate transaminase/alanine transaminase ratio in patients treated with first-line systemic tyrosine kinase inhibitor therapy for metastatic renal cell carcinoma. Int J Urol 25(6):596-603. https://doi.org/10.1111/ iju. 13574

19. Kim SH, Park EY, Joo J, Chung J (2018) The De Ritis and neutrophil-to-lymphocyte ratios may aid in the risk assessment of patients with metastatic renal cell carcinoma. J Oncol 2018:1953571. https://doi.org/10.1155/2018/1953571

20. Ishihara H, Kondo T, Yoshida K, Omae K, Takagi T, Iizuka J et al (2017) Evaluation of preoperative aspartate transaminase/ alanine transaminase ratio as an independent predictive biomarker in patients with metastatic renal cell carcinoma undergoing cytoreductive nephrectomy: a propensity score matching study. Clin Genitourin Cancer 15(5):598-604. https://doi.org/10.1016/j. clgc.2017.04.011

21. Sekar RR, Patil D, Baum Y, Pearl J, Bausum A, Bilen MA et al (2017) A novel preoperative inflammatory marker prognostic score in patients with localized and metastatic renal cell carcinoma. Asian J Urol 4(4):230-238. https://doi.org/10.1016/j. ajur.2017.04.002

22. Sella A, Michaelson MD, Matczak E, Simantov R, Lin X, Figlin RA (2017) Heterogeneity of patients with intermediate-prognosis metastatic renal cell carcinoma treated with sunitinib. Clin Genitourin Cancer 15(2):291-9.e1. https://doi.org/10.1016/j. clgc.2016.08.013

23. Iacovelli R, De Giorgi U, Galli L, Zucali P, Nole F, Sabbatini $\mathrm{R}$ et al (2018) Is it possible to improve prognostic classification in patients affected by metastatic renal cell carcinoma with 
an intermediate or poor prognosis? Clin Genitourin Cancer 16(5):355-9.e1. https://doi.org/10.1016/j.clgc.2018.04.007

24. Bezan A, Mrsic E, Krieger D, Stojakovic T, Pummer K, Zigeuner $R$ et al (2015) The preoperative AST/ALT (De Ritis) ratio represents a poor prognostic factor in a cohort of patients with nonmetastatic renal cell carcinoma. J Urol 194(1):30-35. https://doi. org/10.1016/j.juro.2015.01.083

25. Canat L, Ataly HA, Agalarov S, Alkan I, Altunrende F (2018) The effect of AST/ALT (De Ritis) ratio on survival and its relation to tumor histopathological variables in patients with localized renal cell carcinoma. Int Braz J Urol 44(2):288-295. https://doi. org/10.1590/s1677-5538.Ibju.2017.0173
26. McKay RR, Kroeger N, Xie W, Lee JL, Knox JJ, Bjarnason GA et al (2014) Impact of bone and liver metastases on patients with renal cell carcinoma treated with targeted therapy. Eur Urol 65(3):577-584. https://doi.org/10.1016/j.eururo.2013.08.012

27. Ljungberg B, Albiges L, Bensalah K, Bex A, Giles R, Hora M et al (2019) EAU guideline for renal cell carcinoma. https://urowe b.org/guideline/renal-cell-carcinoma/. Accessed 03/26/2020

Publisher's Note Springer Nature remains neutral with regard to jurisdictional claims in published maps and institutional affiliations. 